\title{
Stability Analysis of a SEIV Epidemic Model with Saturated Incidence Rate
}

\author{
O. Adebimpe 1* $^{*}$ \\ ${ }^{I}$ Department of Mathematics and Physical Science, Osun State University, Oshogbo, Nigeria.
}

Article Information

DOI: 10.9734/BJMCS/2014/2758

Editor $(s)$ :

(1) Narayan Thapa, Department of Mathematics and Computer Science, Minot State University, Minot, USA (2) Paul Bracken, Department of Mathematics, The University of Texas-Pan American Edinburg, TX 78539, USA. (3) Tian-Xiao He, Department of Mathematics and Computer Science, Illinois Wesleyan University, USA.

Reviewers:

(1) Anonymous, Beijing Normal University, China. (2) Anonymous, Hunaghuai University, China. (3) Anonymous, Xi'an Jiaotong University, China. Peer review History: http://www.sciencedomain.org/review-history.php?iid=669\&id=6\&aid=6300

\begin{abstract}
In this paper, a SEIV epidemic model with saturated incidence rate that incorporates polynomial information on current and past states of the disease is investigated. The model exhibits two equilibria, disease-free equilibrium (DFE) and the endemic equilibrium (EE). It is shown that if the basic reproduction number, $\mathrm{R}_{0}<1$, the DFE is locally asymptotically stable and by the use of Lyapunov function, DFE is globally asymptotically stable and in such a case, the EE is unstable. Moreover, if $\mathrm{R}_{0}>1$, the endemic equilibrium is locally asymptotically stable. The effects of the rate at which vaccine wanes $(\omega)$ are investigated through numerical stimulations.

Keywords: SEIV epidemic model, saturated incidence rate, basic reproduction number, locally and globally stable.
\end{abstract}

\section{Introduction}

Vaccinating susceptible against disease infections is an effective measure to control and prevent the spread of the infection. [1] investigated SIS model with vaccination, standard incidence and no disease-induced deaths. In [2], they formulated an SIRS model with vaccinations standard incidence and no disease-induced deaths. [3] studied an SIS model with vaccination, standard incidence and the disease induced death. [4,5] all analyzed global behaviour of simple SIS

*Corresponding author: olukayode.adebimpe @uniosun.edu.ng; 
vaccination epidemic models under the condition that the vaccine is perfectly efficient. In [6], they introduced a vaccination compartment into an SIS model. They assumed that in vaccine takes effect in duration of length $\tau$.

In [7], they studied an SEIV epidemic model with vaccination and vertical transmission. Their results were written in form of basic reproduction number, $R_{0}$ and they carried out a bifurcation analysis and obtain the conditions ensuring that the system exhibits backward bifurcation. [8] extended the model in [9] by incorporating important practical factors as the proportion of recruited individuals that are exposed or infectious, the recovery rate of exposed individuals and the mortality rate due to the infection. In [10], he discussed a vaccination model with non-linear incidence rate and vaccination waning period like [9] but they numerically simulated the model to see how variations in rate of the variables. In [11], they introduced a saturated incidence rate $g$ (I) $\mathrm{S}$ into epidemic models, where $\mathrm{g}(\mathrm{I})$ tends to a saturation level when $\mathrm{I}$ gets large, i.e. $g(I)=\frac{k I}{1+\alpha I}$ where kI measures the infection force of the disease and $\frac{1}{1+\alpha I} \quad$ measures the inhibition effect from the behavioural change of the susceptible individuals when their number increases or from the crowding effect of the infective individual. In this paper, we extend the work done by [9] to incorporate a saturated incidence rate as used by [11].

$$
\begin{aligned}
& \frac{d S}{d t}=(1-\rho) \pi-\frac{\beta S I}{1+\alpha I}-\mu S+\omega V \\
& \frac{d E}{d t}=\frac{\beta S I}{1+\alpha I}-(\mu+\sigma) E \\
& \frac{d I}{d t}=\sigma E-(\mu+\gamma) I \\
& \frac{d V}{d t}=\rho \pi-\mu V+\gamma I-\omega V
\end{aligned}
$$

where

$\pi=$ recruitment of individuals that includes new born and immigrants into the susceptible population $\mathrm{S}, \rho=$ fraction of recruited individuals who are vaccinated, $\beta=$ rate at which susceptible individual become infected by those who are infectious, $\mu=$ natural death rate, $\sigma=$ rate at which exposed individuals become infectious so that $1 / \sigma$ is the mean latent period. $\gamma=$ rate at which infected individuals are treated or recovered, $\frac{1}{1+\alpha(I)}=$ measurement of psychological or inhibition effect of the behavioural change of the susceptible individuals when there is an increase in the number of infective individuals, $\omega=$ rate at which vaccine wanes. The total population size $\mathrm{N}(\mathrm{t})$ can be determined by $N(t)=S(t)+E(t)+I(t)+V(t)$, and is a solution of the differential equation 


$$
\frac{d N(t)}{d t}=\pi-\mu N(t)
$$

Therefore, in the rest of the paper, we will study (1.1) in the closed set

$$
D=\left\{\left(S(t), E(t), I(t), V(t) \in R_{+}^{4} \mid S(t)+E(t)+I(t)+V(t) \leq \frac{\pi}{\mu}\right\}\right.
$$

\section{The Basic Reproduction Number}

It is easy to see that the region $\{(S, E, I, V), S>0, I \geq 0, E \geq 0, V>0\}$ is positively invariant for the model (1.1). Summing up the four equations in model (1.1), we have

$$
\frac{d}{d t}(S+E+I+V)=\mu\left[\frac{\pi}{\mu}-(S+E+I+V)\right] .
$$

Then, $\quad \lim _{t \rightarrow \infty} \sup (S+E+I+V) \leq \frac{\pi}{\mu}$. So, we study the dynamic behaviour of model (1.1) on the region

$$
\Sigma=\left\{(S, E, I, V) \quad S>0, I \geq 0, E \geq 0, V>0, S+E+I+V \leq \frac{\pi}{\mu}\right\},
$$

which is a positive invariant set for (1.1)

Corresponding to $\mathrm{E}=\mathrm{I}=0$, model (1.1) always has a disease-free equilibrium,

$$
P_{0}=\left(\frac{(\mu+\omega-\rho \mu) \pi}{\mu(\mu+\omega)}, \quad 0, \quad 0, \frac{\rho \pi}{\mu+\omega}\right)
$$

Let $x=(S, E, I, V)^{T}$.Then the model (1.1) can be written as $\frac{d x}{d t}=F(x)-V(x)$

where $F(x)=\left(\begin{array}{c}\beta S I \\ \hline 1+\alpha I \\ 0 \\ 0 \\ 0\end{array}\right), \quad V(x)=\left(\begin{array}{c}\mu E+\sigma E \\ \gamma+\mu I-\sigma E \\ -(1-\rho) \pi+\frac{\beta S I}{1+\alpha I}-\omega V+\mu S \\ \mu V+\omega V-\rho \pi-\gamma\end{array}\right)$ 
We have

$$
\begin{array}{ll}
F=\left(\begin{array}{cc}
0 & \beta S_{0} \\
0 & 0
\end{array}\right) \quad V=\left(\begin{array}{cc}
\mu+\sigma & 0 \\
-\sigma & \mu+\gamma
\end{array}\right) \\
\text { So, } \quad V^{-1}=\left(\begin{array}{cc}
\frac{1}{\mu+\sigma} & 0 \\
\frac{\sigma}{(\mu+\sigma)(\mu+\gamma)} & \frac{1}{\mu+\gamma}
\end{array}\right)
\end{array}
$$

$$
F V^{-1}=\left(\begin{array}{cc}
\frac{\sigma \beta S_{0}}{(\mu+\sigma)(\mu+\gamma)} & \frac{\beta S_{0}}{(\mu+\gamma)} \\
0 & 0
\end{array}\right)
$$

In [12], the basic reproduction number is defined as the spectral radius of the next generation matrix

$$
F V^{-1}\left(\rho\left(F V^{-1}\right)\right)
$$

So, according to Theorem (2) in [12], the basic reproduction number of model (1.1), is

$$
R_{0}=\ell\left(F V^{-1}\right)=\frac{\sigma \beta S_{0}}{(\mu+\sigma)(\mu+\gamma)}
$$

where $S_{0}=\frac{(\mu+\sigma)(1-\rho) \pi+\omega \rho \pi}{\mu(\mu+\omega)}$

Therefore,

$$
R_{0}=\frac{\sigma \beta[(\mu+\omega)(1-\rho) \pi+\omega \rho \pi]}{\mu(\mu+\omega)(\mu+\sigma)(\mu+\gamma)}
$$

Note: The spectral radius $\left(\rho\left[F V^{-1}\right]\right)$ is the dominant eigenvalue of the matrix $\mathrm{FV}^{-1}$. 


\section{Local and Global Stability of Disease Free Equilibrium}

$$
\text { Let } x=S-S_{0}, \quad I=I, E=E, \quad y=V-V_{0}
$$

Then, equation (1.1) becomes

$$
\begin{aligned}
\frac{d S}{d t}= & (1-\rho) \pi-\beta I\left(x+\frac{(\mu+\omega)(1-\rho) \pi+\omega \rho \pi}{\mu(\mu+\omega)}\right)(1+\alpha I)^{-1}- \\
& \mu\left(x+\frac{(\mu+\omega)(1-\rho) \pi+\omega \rho \pi}{\mu(\mu+\omega)}\right)+\omega\left(y+\frac{\rho \pi}{\mu+\omega}\right) \\
\frac{d E}{d t}= & \beta I\left(x+\frac{(\mu+\omega)(1-\rho) \pi+\omega \rho \pi}{\mu(\mu+\omega)}\right)(1+\alpha I)^{-1}-(\mu+\sigma) E \\
\frac{d I}{d t}= & \sigma E-(\mu+\gamma) I \\
\frac{d V}{d t}= & \rho \pi-(\mu+\omega)\left(y+\frac{\rho \pi}{\mu+\omega}\right)+\gamma I
\end{aligned}
$$

The Jacobian matrix of equation (3.2) after linearization is

$$
A=\left(\begin{array}{cccc}
-\mu & 0 & \frac{-\beta[(\mu+\omega)(1-\rho) \pi+\omega \rho \pi]}{\mu(\mu+\omega)} & \omega \\
0 & -(\mu+\sigma) & \frac{\beta[(\mu+\omega)(1-\rho) \pi+\omega \rho \pi]}{\mu(\mu+\omega)} & 0 \\
0 & \sigma & -(\mu+\gamma) & 0 \\
0 & 0 & \gamma & -(\mu+\omega)
\end{array}\right)
$$

Let $\frac{\beta[(\mu+\omega)(1-\rho) \pi+\omega \rho \pi]}{\mu(\mu+\omega)}=\Gamma$

$$
|A-\lambda I|=\left|\begin{array}{cccc}
-(\mu+\lambda) & 0 & -\Gamma & \omega \\
0 & -(\mu+\sigma+\lambda) & \Gamma & 0 \\
0 & \sigma & -(\mu+\gamma+\lambda) & 0 \\
0 & 0 & \gamma & -(\mu+\omega+\lambda)
\end{array}\right|=0
$$

Therefore, $\lambda_{1}=-\mu, \lambda_{2}=-(\mu+\omega)$, and the roots of the quadratic

$$
\lambda^{2}+(2 \mu+\sigma+\gamma) \lambda+\mu^{2}+\mu \gamma+\sigma \mu+\sigma \gamma-\sigma \Gamma=0
$$


For negative roots, we must have by Descartes' rule of signs

$$
\mu^{2}+\mu \gamma+\sigma \mu+\sigma \gamma-\sigma \Gamma>0
$$

That is,

$$
\begin{aligned}
& (\mu+\sigma)(\mu+\gamma)>\sigma \beta \frac{[(\mu+\omega)(1-\rho) \pi+\omega \rho \pi]}{\mu(\mu+\omega)} \\
& \sigma \beta \frac{[(\mu+\omega)(1-\rho) \pi+\omega \rho \pi]}{\mu(\mu+\omega)(\mu+\sigma)(\mu+\gamma)}<1
\end{aligned}
$$

Therefore,

$$
R_{0}=\frac{\sigma \beta[(\mu+\omega)(1-\rho) \pi+\omega \rho \pi]}{\mu(\mu+\omega)(\mu+\sigma)(\mu+\gamma)}<1
$$

Theorem 3.1: If $\mathrm{R}_{0}<1$, the disease free equilibrium $\mathrm{P}_{0}$ is locally asymptotically stable; if $\mathrm{R}_{0}=1$, $\mathrm{P}_{0}$ is stable; if $\mathrm{R}_{0}>1, \mathrm{P}_{0}$ is unstable.

Proof: We shall check the stability of the disease-free equilibrium $\mathrm{P}_{0}$, from the model, thus the linearization of model of the disease-free equilibrium $\mathrm{P}_{0}$ gives the following characteristics equation.

$$
-(\mu+\lambda)(\mu+\omega+\lambda)\left[(\mu+\gamma+\lambda)(\mu+\sigma+\lambda)-\frac{\sigma \beta[(\mu+\omega)(1-\rho) \pi+\omega \rho \pi]}{\mu(\mu+\omega)}\right]=0
$$

Thus, we have

$$
-(\mu+\lambda)(\mu+\omega+\lambda)\left[\lambda^{2}+(2 \mu+\gamma+\sigma) \lambda+\mu^{2}+\mu \gamma+\sigma \mu+\sigma \gamma-\frac{\sigma \beta[(\mu+\omega)(1-\rho) \pi+\omega \rho \pi]}{\mu(\mu+\omega)}\right]=0
$$

From equation (3.4), it can be seen that $\lambda_{1}=-\mu, \lambda_{2}=-(\mu+\omega)$ are two of the eigenvalues and they are always negative. To obtain the other eigenvalues of equation (3.4) we consider the equation

$$
\lambda^{2}+(2 \mu+\gamma+\sigma) \lambda+\mu^{2}+\mu \gamma+\sigma \mu+\sigma \gamma-\frac{\sigma \beta[(\mu+\omega)(1-\rho) \pi+\omega \rho \pi]}{\mu(\mu+\omega)}=0
$$

From equation (3.5) we see that all roots have negative real parts if

$$
\mu^{2}+\mu \gamma+\sigma \mu+\sigma \gamma-\frac{\sigma \beta[(\mu+\omega)(1-\rho) \pi+\omega \rho \pi]}{\mu(\mu+\omega)}>0
$$


That is, if $\mathrm{R}_{0}<1$.

The disease-free equilibrium $\mathrm{P}_{0}$, is locally asymptotically stable.

If $R_{0}=1$, one eigenvalue of equation (3.5) is zero and it is simple. Then $\mathrm{P}_{0}$ is stable. If $\mathrm{R}_{0}>1$, one of the roots of equation (3.5) has a positive real part, then $\mathrm{P}_{0}$ is unstable.

Theorem 3.2: If $R_{0}<1$, the disease-free equilibrium $P_{0}$ is globally asymptotically stable in $D$.

Proof: Consider the Lyapunov function $L(t)=\sigma E+(\mu+\sigma) I$. Its derivative along the system (1.1) is

$$
\begin{aligned}
& L^{1}(t)=\frac{\sigma \beta S_{0} I}{1+\alpha I}-(\mu+\sigma)(\mu+\gamma) I \\
& L^{1}(t)=\left[\frac{\sigma \beta S_{0}}{(1+\alpha I)(\mu+\sigma)(\mu+\gamma)}-1\right] I \\
& L^{1}(t)=\left[\frac{R_{0}}{(1+\alpha I)}-1\right]
\end{aligned}
$$

If $R_{0}<1, I \neq 0, L^{1}(t)<0$

Therefore, disease-free equilibrium is globally asymptotically stable

\section{Local Stability of the Endemic Equilibrium}

It is a known fact that the disease is endemic if the infectious part of the population persists above a certain positive level for sufficiently large time. The disease is endemic if (1.1) is uniformly persistent as in [13].

Theorem 1: $R_{0}>1$, then unique endemic equilibrium $P_{*}$ of (1.1) is locally asymptotically stable in D.

Proof: The method of Routh-Hurwitz will be used to show the local asymptotic stability of the equilibrium, $\mathrm{P}_{*}$. The Jacobian matrix of (1.1) at a point $D=(S(t), E(t), I(t), V(t))$ is

$$
J(P)=\left[\begin{array}{cccc}
-\left(\beta I_{*}+\mu\right) & 0 & -\beta S_{*} & \omega \\
\beta I_{*} & -(\mu+\sigma) & \beta S_{*} & 0 \\
0 & \sigma & -(\mu+\gamma) & 0 \\
0 & 0 & \gamma & -(\mu+\omega)
\end{array}\right]\left(\begin{array}{l}
x \\
y \\
z \\
q
\end{array}\right)+\text { nonlinear terms }
$$


It is proved that the matrix $\mathrm{P}$ is stable with all its eigenvalues have negative real parts. From the Jacobian matrix, the characteristics equation is

$$
\lambda^{3}+a_{1} \lambda^{2}+a_{2} \lambda+a_{3}=0
$$

where

$$
\begin{aligned}
& a_{1}=\sigma+\gamma+3 \mu+\beta I_{*}>0 \\
& a_{2}=2 \beta \mu I_{*}+\beta \sigma I_{*}+\beta I_{*}+3 \mu^{2}+2 \mu \sigma+2 \mu \gamma+\sigma \gamma-\sigma \beta S_{*} \\
& a_{3}=\beta \mu^{2} I_{*}+\beta \mu I_{*}+\beta \sigma \mu I_{*}+\beta \sigma I_{*}+\mu^{3}+\mu^{2} \gamma+\mu^{2} \sigma+\mu \sigma \gamma-\sigma \beta \mu S_{*}-\omega \sigma \beta \gamma I_{*}
\end{aligned}
$$

If $a_{1} a_{2}>a_{3}$ the endemic equilibrium $\mathrm{P}_{*}$ is locally asymptotically stable in $\mathrm{D}$.

\section{Numerical Simulation}

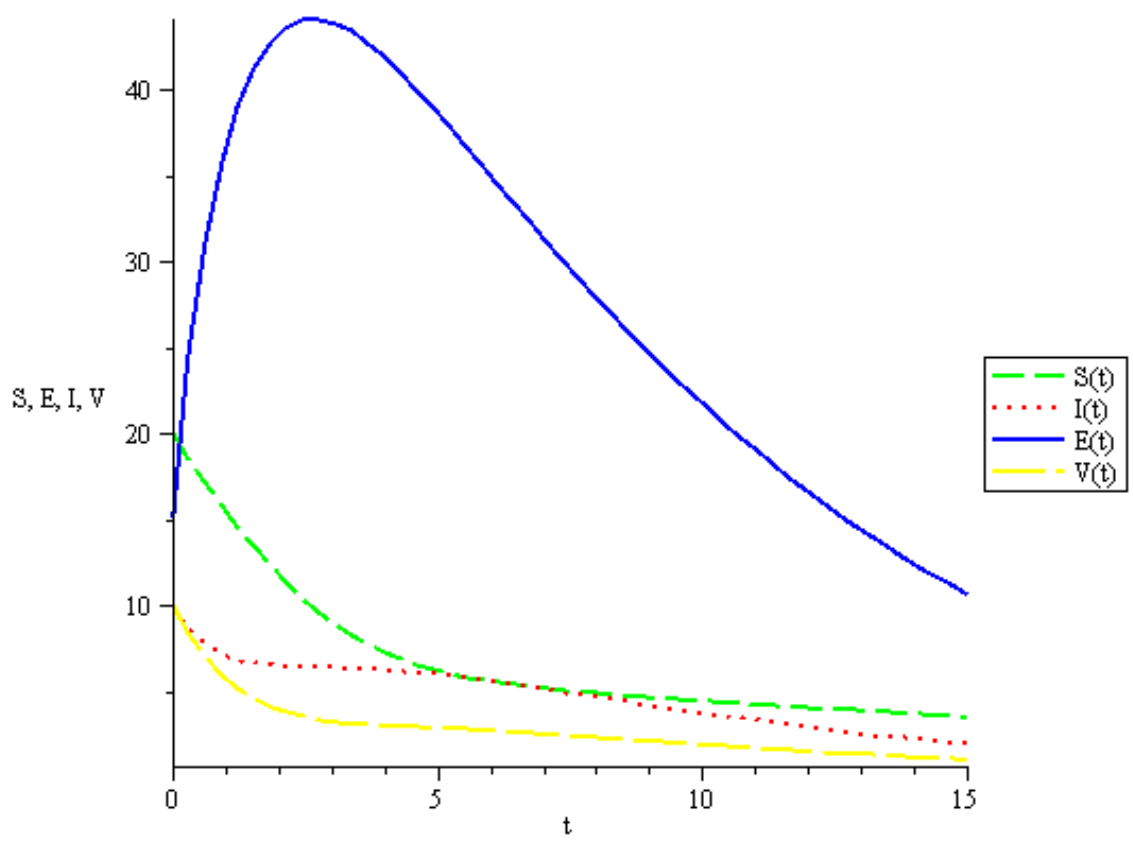

Fig. 5.1. Graph of $\mathbf{S}(\mathbf{t}), \mathbf{E}(\mathbf{t}), \mathbf{I}(\mathbf{t}), \mathbf{V}(\mathbf{t})$ against time (t) when $\rho=0.1, \pi=0.2, \beta=0.1$, $\alpha=0.1, \mu=0.2, \omega=0.9, \sigma=0.1, \gamma=0.5$ 


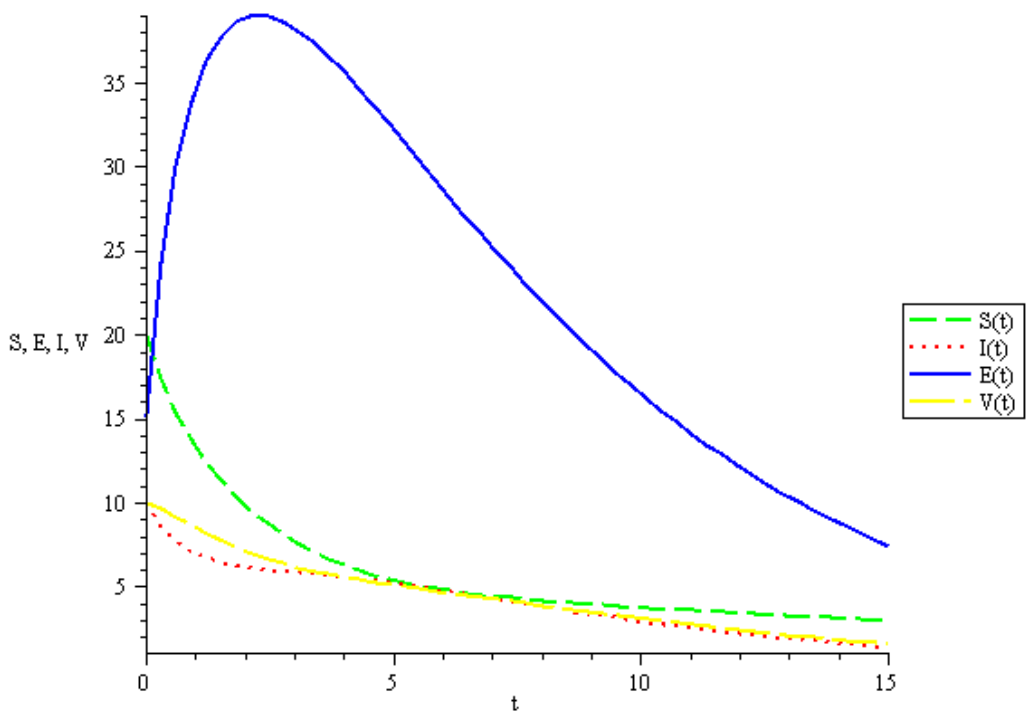

Fig. 5.2. Graph of $\mathbf{S}(\mathbf{t}), \mathbf{E}(\mathbf{t}), \mathbf{I}(\mathbf{t}), \mathbf{V}(\mathbf{t})$ against time (t) when $\rho=0.1, \pi=0.2, \beta=0.1$, $\alpha=0.1, \mu=0.2, \omega=0.4, \sigma=0.1, \gamma=0.5$

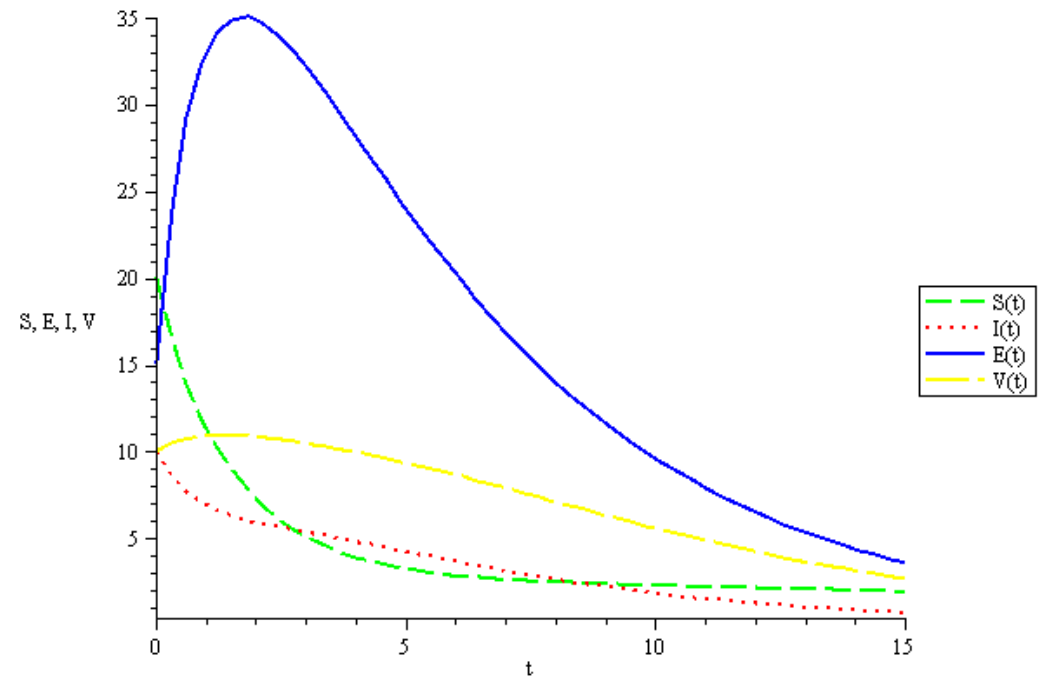

Fig. 5.3. Graph of $\mathbf{S}(\mathbf{t}), \mathbf{E}(\mathbf{t}), \mathbf{I}(\mathbf{t}), \mathbf{V}(\mathbf{t})$ against time $(\mathbf{t})$ when $\rho=0.1, \pi=0.2$, $\beta=0.1, \alpha=0.1, \mu=0.2, \omega=0.1, \sigma=0.1, \gamma=0.5$ 


\section{Conclusions and Discussion}

This paper has considered a SEIV model that incorporates an incidence rate of the form $\frac{\beta S I}{1+\alpha I}$.

We discussed the local and global stabilities of the disease-free equilibrium and as well as the local stability of the endemic equilibrium. We presented the results in the form of basic reproduction number, $\mathrm{R}_{0}$.

The effects at which vaccine wanes were investigated and it is discovered in Fig. 5.1, Fig. 5.2 and Fig. 5.3 that the smaller the value that represents the rate at which vaccine wanes $\omega$, the greater the effect on the model. This implies that vaccination has a role to play in any disease eradication and that the rate at which vaccine wanes should be reduced to the barest minimum for the vaccine to be effective in the population.

\section{Acknowledgement}

The author acknowledged the support received from Institute of Mathematical Sciences (IMS), National University of Singapore (NUS), Singapore during his 2-month stay at the institute and also thank the anonymous referees for their useful feedback and helpful comments and suggestions which have improved the manuscript. The author wishes to dedicate the research work to the memory of his late supervisor and mentor, Professor Reuben Olafenwa Ayeni.

\section{Competing Interests}

Author has declared that no competing interests exist.

\section{References}

[1] Kribs-Zaleta CM, Velasco-Hernandez JK. A simple vaccination model with multiple endemic states. Math Biosci. 2000;164:183-201.

[2] Arino J, Mccluskey CC, van den Driessche P. Global Results for an epidemic model with vaccination that exhibits backward bifurcation. SIAM J Appl Math. 2003;64:260-76.

[3] Li J, Ma, Z, Zhou Y. Global Analysis of SIS epidemic model with a simple vaccination and multiple endemic equilibria. Acta Math Sci. 2006;26B:83-93.

[4] Li J, Ma Z. Qualitative analyzes of SIS epidemic model with vaccination and varying total population size. Math Comp Model. 2002;35:1235-243.

[5] Li J, Ma Z. Global analysis of SIS epidemic models with variable total population size. Math Comput Model. 2004b;39:1231-1242. 
[6] Li J, Ma Z. Stability analysis for SIS epidemic model with vaccination and constant population size. Disc Cont Dyn. Sys. 2004;B4:637-44.

[7] Long D, Xiang Z. On the study of an SEIV epidemic model concerning vaccination and vertical transmission. 2011;1(1):21-30.

[8] Chika M, Ezeofor OV. Extension and modification of an SEIV vaccination model. Journal of the Nigerian Mathematical Society. 2011;30:163-77.

[9] Cai L, Li X. Analysis of a SEIV epidemic model with a non-linear incidence rate. Applied Mathematical Modelling. 2009;33:2919-926.

[10] Abdulrazak AJ, Ibrahim MO, Usman IO. A Seiv Vaccination Model with General NonLinear Incidence Rate and Wanning Preventive Vaccine. IOSR Journal of Mathematics. 2012;4(2):44-51.

[11] Capasso V, Serio G. A generalization of the Kermack-Mckendrick deterministic epidemic model. Math. Biosci. 1978;42:43-61.

[12] Van den Driessche P, Watmough J. Reproduction numbers and sub- threshold endemic equilibria for compartmental models of disease transmission. Math. Biosci. 2002;180:2948 .

[13] Butler GJ, Waltman P. Persistence in Dynamical Systems. J. Differential Equations. 1986;63:225-63.

(C) 2014 Adebimpe; This is an Open Access article distributed under the terms of the Creative Commons Attribution License (http://creativecommons.org/licenses/by/3.0), which permits unrestricted use, distribution, and reproduction in any medium, provided the original work is properly cited.

Peer-review history:

The peer review history for this paper can be accessed here (Please copy paste the total link in your browser address bar)

www.sciencedomain.org/review-history.php?iid=669\&id=6\&aid=6300 